Post-print of: Morillo, M. et al. "Superparamagnetic iron oxide nanoparticles-loaded polyacrylonitrile nanofibers with enhanced arsenate removal performance" in Environmental Science Nano, núm. 3 (2016), p. 1165-1173. The final version is available at DOI 10.1039/c6en00167j

Received 00th January 20xx, Accepted 00th January 20xx

DOI: $10.1039 / \times 0 x \times 00000 x$

www.rsc.org/

\section{Superparamagnetic iron oxide nanoparticles-loaded polyacrylonitrile nanofibers with enhanced arsenate removal performance}

\author{
D. Morillo, ${ }^{a}$ M. Faccini ${ }^{a}$, D. Amantia ${ }^{a}$, G. Pérez $^{b}$, M. Valiente $^{b}$ and L. Aubouy ${ }^{a}$
}

\section{Introduction}

Arsenic contamination in natural water due to increased population and industrial activities is a global threat to both human health and the environment. Mining wastes, petroleum refining, sewage sludge, agricultural chemicals, ceramic manufacturing industries, and coal fly ash are some of the anthropogenic activities that increase arsenic concentrations in surface water as well as groundwater. ${ }^{1}$ Natural phenomena such as weathering, erosion of rocks/soils and volcanic emissions also contribute arsenic in aqueous system. $^{2}$

Due to its high toxicity ${ }^{3}$ and carcinogenicity even at very low concentrations, $^{4-8}$ the World Health Organization (WHO) has reduced the Maximum Contamination Level (MCL) from $50 \mu \mathrm{g} / \mathrm{L}$ to $10 \mu \mathrm{g} / \mathrm{L}$. Therefore, it is noteworthy that the continuous

\footnotetext{
a. LEITAT Technological Center, C/ Pallars, 179-185, 08005 Barcelona, Spain

${ }^{b .}$ Universitat Autònoma de Barcelona, Centre GTS, Department of Chemistry, 08193 Bellaterra (Barcelona), Spain.

Electronic Supplementary Information (ESI) available: [Electronic supplementary Information (ESI) available: Electrospinning conditions, experimental set-up for adsorption process in continuous flow mode, lixiviation water characterization, pictures of SPION loaded nanofibers, nanofibers size diameter distribution diagrams, FTIR spectra, swelling effect of HPAN-10-SPION, pictures of the swelling effect, adsorption capacity for the different HPAN nanofibers without SPION. See DOI: $10.1039 / x 0 x x 00000 x$
}

strengthening regulations make necessary the improvement of existing arsenic remediation methods. ${ }^{9,10}$

Although multiple water purification methods such as precipitation, ${ }^{11}$ ion exchange ${ }^{7}$ or membrane processes ${ }^{12}$ have been studied for arsenic removal, selective adsorption ${ }^{13}$ from solution has received more attention and it is considered to be one of the most promising approaches due to a simple assembly, easy operation, low cost and high efficiency.

Iron oxide-based materials have been widely used in arsenic removal because of their low cost, and natural abundance. Iron oxide nanoparticles provides an advantage due to an increase of the surface-volume ratio and specific surface area, allowing more active sites to better improve the adsorption process. ${ }^{14}$ Previous studies show that iron oxide nanoparticles (magnetite and maghemite) with sizes between 3.8 and $12 \mathrm{~nm}$ exhibits significantly increased arsenic adsorption capacities compared with bulk iron oxides, which may result from more adsorption sites being exposed to arsenic species. ${ }^{15,16}$

However, nanoparticles tend to easily aggregate into large particles, leading to deteriorated adsorption performance. Besides, it is difficult to practically apply nanoparticles in the wastewater treatment because small particles may cause difficulties in separation. ${ }^{17}$ Ensuring the absence of nanoscale solids in the purified streams becomes critical for nanotechnology based 
products to comply with the strict environmental regulations. Nanoparticles released during the treatment process may pose a health risk, as the toxicity effect to the end user is not well known. ${ }^{18}$

To avoid these problems, the dispersion and fixation of iron oxide nanoparticles over porous supports has been a common strategy in the development of safe and efficient arsenic adsorbents. ${ }^{19-22}$ Among the several available supporting materials, electrospun polymeric nanofibers present high surface area able to interact easily with the inorganic particles and improve the contact time with the contaminated media. Electrospinning is a well-established and versatile process that has been used to produce ultrafine fibers including microfibers $(>1 \mu \mathrm{m})$ or nanofibers $(<1000 \mathrm{~nm}) .{ }^{23,24}$ The main advantage of the electrospinning process among other techniques is the relative quick, simple, and economical way to fabricate a variety of materials into nanofibrous structures. ${ }^{25}$ Polyacrylonitrile (PAN) nanofibers, because of their availability as commodity textile materials, have been modified extensively and easily by several processes (chemical grafting, wet chemistry, particles incorporation, etc...) to achieve a certain affinity for metal ion removal. ${ }^{26}$

In the present work, polyacrylonitrile (PAN) nanofibers obtained by electrospinning process were chosen as porous support for superparamagnetic iron oxide nanoparticles (SPION). SPION were loaded onto polymeric nanofibers by a straight-forward impregnation method and tested for the efficient and selective adsorption of arsenate from water. The influence of both nanofibers diameter size and SPION load on the sorption properties were first studied in batch mode. The optimal nanostructured adsorbent was tested in a column in continuous flow operation mode using real wastewater matrix in presence of a large amount of competing anions and interfering cations. No release of nanoparticles during operation was observed.

\section{Experimental section}

\section{Chemicals and Reagents}

Iron(III) chloride hexahydrate, iron(II) chloride anhydrous, ammonium hydroxide, sodium acetate trihydrate, acetic acid, sodium hydrogen arsenate heptahydrate and hydrochloric acid were used for the SPION synthesis. Polyacrylonitrile PAN ( $\mathrm{Mw}=150.000 \mathrm{~g} / \mathrm{mol}$, powder) and $\mathrm{N}-\mathrm{N}$ dimethylformamide (DMF) were used for the electrospinning solution. Tetramethyl ammonium hydroxide (TMAOH, Fluka 25\%) was used as dispersant agent and high purity water with a resistivity of $18 \mathrm{M} \Omega / \mathrm{cm}$ was used throughout all the experiments. All chemicals and reagents were purchased by Sigma-Adrich.

\section{Synthesis of SPION}

The synthesis of SPION was performed as described elsewhere ${ }^{27}$ with some modifications to obtain a high reaction yields. The synthesis requires a constant bubbling of nitrogen during the reaction to prevent oxidation of $\mathrm{Fe}^{2+}$ to $\mathrm{Fe}^{3+}$ and therefore, the generation of other iron oxides such as maghemite or ferrihydrite. A $0.5 \mathrm{~mol} / \mathrm{L}$ of $\mathrm{Fe}^{3+}$ solution in a chloride medium was prepared by dissolving $\mathrm{FeCl}_{3} \cdot 6 \mathrm{H}_{2} \mathrm{O}$ in $0.2 \mathrm{M} \mathrm{HCl}$ solution. $\mathrm{A}$ 0.7 $\mathrm{M} \mathrm{NH}_{4} \mathrm{OH}$ solution was heated to $70{ }^{\circ} \mathrm{C}$. Later on, $\mathrm{Fe}^{3+}$ solution was added to the $\mathrm{NH}_{4} \mathrm{OH}$ solution. After a few minutes, anhydrous $\mathrm{FeCl}_{2}$ was added, in a ratio $1 / 2$ of $\mathrm{Fe}^{2+} / \mathrm{Fe}^{3+}$. Then, the solution was kept 45 minutes under mechanical stirring for the nanoparticles ageing. After sample cooling, the resulting suspension was centrifuged at $2000 \mathrm{rpm}$ and the nanoparticles were separated with the help of a magnet and washed with high purity water several times. Subsequent dispersion step in $0.01 \mathrm{M} \mathrm{TMAOH}$ solution allows obtaining SPION in a stable suspension for 6-8 months under nitrogen atmosphere.

\section{Synthesis of electrospun PAN nanofibers}

Solutions for electrospinning were prepared by dissolving PAN at concentrations ranging from 7 to $15 \mathrm{wt} \%$ in DMF at $60{ }^{\circ} \mathrm{C}$. All polymer solutions were mixed by a magnetic stirrer for a sufficient time until they became homogeneous. The viscosity and electrical conductivity of polymer solutions were measured by a digital viscometer (DV-E, Brookfield Co.) and an electric conductivity meter (CRISON EC-meter BASIC) at $25{ }^{\circ} \mathrm{C}$. To produce nanofibers, the solutions were electrospun onto an aluminum foil by using commercially available electrospinning equipment (MECC Co. LTD., model NF-103). Plastic syringes fitted with metal needles were used as electrospinning nozzles. Typical operating conditions were: flow rates of $1-2 \mathrm{~mL} / \mathrm{h}$, applied voltages between 20 and $30 \mathrm{kV}$, and working distance of $15 \mathrm{~cm}$. Values of viscosity and conductivity of each solution as well as the conditions used for electrospinning are reported in Table S1, ESI. The obtained nanofibers were dried in a vacuum oven for $24 \mathrm{~h}$ at $60{ }^{\circ} \mathrm{C}$ before characterization.

\section{Surface modification of electrospun PAN nanofibers}

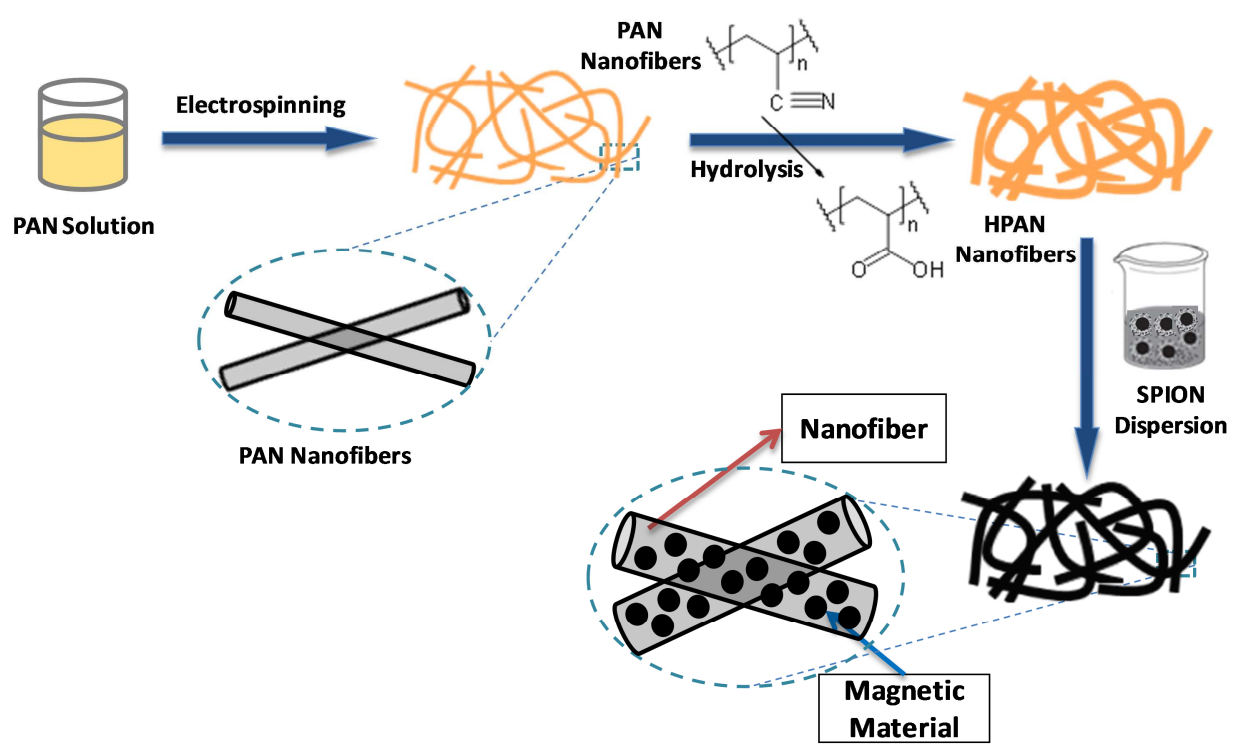

Fig. 1 Scheme of the synthesis process of SPION loaded nanofiber adsorbents. 
Electrospun PAN nanofiber mats $\left(200 \mathrm{~cm}^{2}, 100 \mathrm{mg}\right.$ approximately) were immersed in $100 \mathrm{~mL}$ of 15 wt $\% \mathrm{NaOH}$ aqueous solution for 60 $\min$ at $50^{\circ} \mathrm{C}$. As a result, the nanofiber mat turned yellowish due to the generation of $\mathrm{COO}^{-} \mathrm{Na}^{+}$functional groups. After immersion in a $1.0 \mathrm{M} \mathrm{HCl}$ solution at room temperature for $120 \mathrm{~min}$, the nanofiber sample recovered white appearance, eventually obtaining electrospun hydrolyzed PAN nanofibers (HPAN). ${ }^{28}$ The synthesis process of the SPION loaded HPAN nanofibers is depicted in Fig. 1.

\section{Synthesis of SPION loaded HPAN nanofibers}

HPAN nanofibers (100-150 mg approximately) were immersed in $100 \mathrm{~mL}$ of SPION suspension and kept in contact without stirring for 3 hours at room temperature. Different SPION concentrations were tested to determine the optimal SPION amount with a concentration ranging from 3 to $145 \mathrm{mg} / \mathrm{L}$. Afterwards, the obtained SPION loaded HPAN nanofibers were rinsed with deoxygenated water to remove the SPION excess that is not properly fixed on the nanofiber surface and kept in deoxygenated water for adsorption analysis.

The surface morphology of the nanofiber mats was examined using scanning electron microscopy (SEM) after Gold coating to minimize the charging effect. Images taken by SEM were analyzed to obtain the fiber diameter by the FibraQuant 1.3 software. At least four pictures were used to calculate the mean values of the fiber diameter.

To quantify the amount of SPION loaded over the HPAN nanofibers, $0.5 \mathrm{~g}$ of fibers were treated with $10 \mathrm{~mL}$ of concentrated $\mathrm{HNO}_{3}$ in an analytical microwave digestion system (MARS 5, CEM) following a standard procedure. After digestion, samples were cooled down to room temperature and diluted with a 2 vol $\% \mathrm{HNO}_{3}$ aqueous solution. Iron content was determined by inductively coupled plasma mass spectrometry (ICP-MS, 7500cx, Agilent Technologies).

Nanofiber samples were denoted as PAN- $X$, HPAN- $X$ or HPAN- $X$ SPION where $X$ is the $w t \%$ of PAN in the electrospinning solution.

\section{Materials characterization}

SPION were imaged by high resolution transmission electron microscopy (HR-FEG-TEM, JEOL JEM-2100) to determine the morphology and particle size distribution. The crystallographic phase was undertaken by X-Ray diffraction (XRD, X-Pert Philips diffractometer), using a monochromatized $X$-ray beam with nickel filtered CuK $\alpha$ radiation $(\lambda=0,154021 \mathrm{~nm})$ at standard conditions ( $2 \theta$ range to $10-60^{\circ}$, step size 0.04 and time of step 4 minutes). Magnetic susceptibility of SPION was determined by superconducting quantum Interference device (SQUID, MPMS-XL7, Quantum design $\mathrm{Inc}$ ) in a magnetic field range from -7 to $7 \mathrm{~T}$ at the constant temperature of $300 \mathrm{~K}$, allowing to measure extremely weak magnetic fields based on superconducting loops. SPION loaded HPAN nanofibers were characterized by scanning electron microscope (SEM, Zeiss MERLIN FE, Carl Zeiss Microscopy, LLC) to determine the homogeneity and the nanofiber diameter. Fourier
Transform Infra-red (ATR-FTIR, SPECTROM ON, Perkin-Helmer equipped with ATR diamond at $303 \mathrm{~K}$ ) was used to verify the surface modification of the PAN nanofibers.

\section{Adsorption experiments with SPION loaded HPAN nanofibers in batch mode}

All experiments were performed using $100 \mathrm{mg} / \mathrm{L} \mathrm{As}(\mathrm{V})$ aqueous solutions at $\mathrm{pH}$ between 3.6 and 4.0 , which is reported in literature as the optimal $\mathrm{pH}$ range for the $\mathrm{As}(\mathrm{V})$ adsorption on SPION. ${ }^{29}$

The adsorption process in batch mode was performed by mixing $\mathrm{As}(\mathrm{V})$ solutions in $0.2 \mathrm{~mol} / \mathrm{L}$ Acetic/Acetate media at room temperature with SPION loaded HPAN nanofibers (100 mg) for $1 \mathrm{~h}$ using a rotatory shaker. The $\mathrm{pH}$ of the solutions was controlled using $1.0 \mathrm{M} \mathrm{HNO}_{3}$ or $1.0 \mathrm{M} \mathrm{NaOH}$ solutions and confirmed with $\mathrm{pH}$ measurements ( $\mathrm{pH}$ meter, Crison). After the fixed contact time, the nanofiber mat was removed from the solution which was filtrated with a $0.22 \mu \mathrm{m}$ filter (cellulose acetate, Millipore). The $\mathrm{pH}$ of the solution was measured after adsorption process as the $\mathrm{pH}$ value of the experiment and the arsenic concentration of in solution was determined by ICP-MS.

For each experiment, the adsorption capacity $\left(\mathrm{q}_{\mathrm{As}}, \mathrm{mmol} / \mathrm{g}\right)$ is calculated by measuring, the initial $\left(C_{\text {ini }}, \mathrm{mmol} / \mathrm{L}\right)$ and equilibrium $\left(C_{\text {eq }}, \mathrm{mmol} / \mathrm{L}\right.$ ) arsenic concentration and by applying the following Equation (1):

$$
\mathrm{q}_{\mathrm{As}}=\frac{\mathrm{V}_{\mathrm{ads}} \cdot\left(\mathrm{C}_{\mathrm{ini}}-\mathrm{C}_{\mathrm{eq}}\right)}{\mathrm{m}_{\mathrm{ads}}}
$$

where $V_{a d s}$ is the volume of reaction $(L)$ and $m_{a d s}$ is the adsorbent quantity (g).

\section{Adsorption experiments with SPION loaded HPAN nanofibers in continuous mode}

Adsorption experiments in continuous mode were performed using the setup depicted in Fig. S1, ESI. SPION loaded HPAN nanofibers $(100 \mathrm{mg})$ of were introduced into glass column with a length of 200 $\mathrm{mm}$ and an internal diameter of $15 \mathrm{~mm}$. A peristaltic pump was used to recirculate $2 \mathrm{~L}$ of $5 \mathrm{mg} / \mathrm{L} \mathrm{As}(\mathrm{V})$ solution through the column with a $2 \mathrm{~mL} / \mathrm{min}$ flow rate during 24 hours either by gravity or counterflow mode.

Following the same procedure, experiments simulating real conditions were performed using a complex matrix doped with Arsenic. Specifically, we employed a lixiviate from a dump at $\mathrm{pH} 4$ with high content in $\mathrm{K}^{+}, \mathrm{Na}^{+}, \mathrm{Mg}^{2+}, \mathrm{Ca}^{2+}, \mathrm{Cl}^{-}$and $\mathrm{F}^{-}$doped with 5 $\mathrm{mg} / \mathrm{L} \mathrm{As}(\mathrm{V})$. The complete characterization reported in Table S2, ESI.

\section{Results and discussion}

\section{Characterization of SPION}



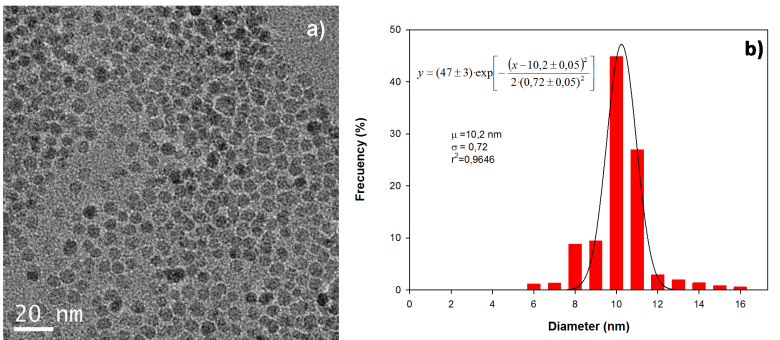

Fig. 2 TEM micrograph of synthesized SPION with its diffractogram (a) and histogram of SPION size distribution (b).

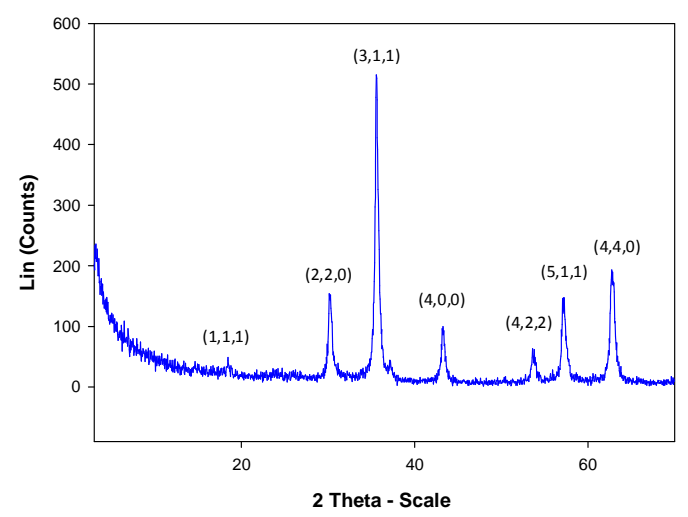

Fig. 3 X-Ray diffraction spectra of SPION.

Nanoparticles morphology, mainly the size, determines the adsorption capacity. TEM micrograph in Fig. 2a shows that the synthesis method employed allowed obtaining nanoparticles with mainly spherical morphology. Although being partially aggregated when in suspension, SPION have a narrow size distribution with an average diameter of $10.2 \mathrm{~nm}$ (as shown in the histogram in Fig. 2b). Such dimension is in agreement with the size reported in literature, 8-10 $\mathrm{nm}$, to reach maximum arsenic adsorption capacity. ${ }^{30-32}$

Additional information regarding SPION crystalline structure is obtained by X-Ray diffraction. The SPION diffractogram shown in Fig. 3 indicates the presence of a single phase corresponding to SPION as compared to a $\mathrm{Fe}_{3} \mathrm{O}_{4}$ standard found in the database. ${ }^{33}$

Superparamagnetic materials have no permanent magnetic moment and, hence, no hysteresis loop. The magnetic susceptibility of SPION powder was determined by assessing its magnetization as a function of magnetic field applied. As shown in Fig. 4, the shape of the hysteresis curve for the sample was normal and tight with no hysteresis losses, typical behavior of a superparamagnet. Under low applied field, a high magnetization (M) value was observed. The saturation magnetization (Ms) and the coercivity $(\mathrm{Hc})$ of the SPION are about $80 \mathrm{emu} / \mathrm{g}$ and 143 Oe respectively, values close to bulk $\mathrm{Fe}_{3} \mathrm{O}_{4}$ (85-100 emu/g and 115-150 Oe, correspondingly). ${ }^{34,35}$ Accordingly to the observed remaining magnetic capacity, together with the high specific surface areas and strong magnetic properties, SPION appears as an excellent adsorbent candidate for environmental applications. ${ }^{36}$

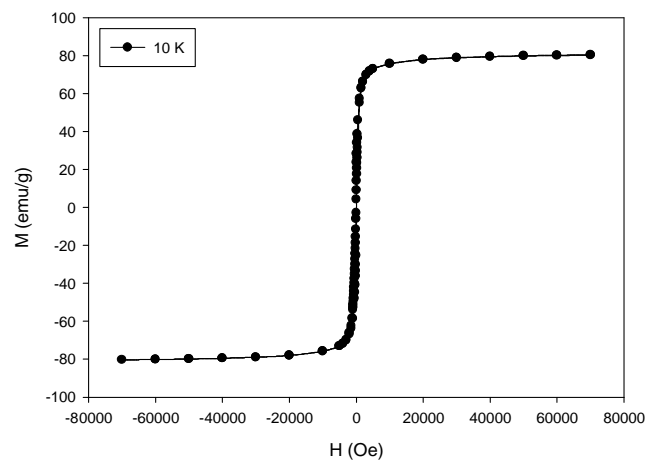

Fig. 4 Magnetic hysteresis loop of SPION.

\section{Characterization of electrospun PAN and HPAN nanofibers}

The morphology of PAN fibers obtained by electrospun polymer solutions at different concentrations and the HPAN obtained after the hydrolysis process was studied by SEM. As Fig. 5 shows, long and highly homogeneous nanofibers without defects were obtained in all cases.

Distribution plots of the nanofiber diameter size determination are presented in Fig S2, ESI. An increase in the PAN concentration in the electrospinning solution produces an increase in the nanofiber size, going from $245 \mathrm{~nm}$ for PAN-7 to $298 \mathrm{~nm}$ for PAN-8.5, $383 \mathrm{~nm}$ for PAN-10 and $2388 \mathrm{~nm}$ for PAN-15. This fact indicates that the fibers morphology is strongly dependent on the polymer concentration, which also affects the viscosity. At higher viscosities there are more chain entanglements and less chain mobility, resulting in less extension during spinning, therefore producing thicker fibers. Moreover, high surface roughness can be appreciated especially in nanofibers with larger diameter.

A comparison between samples before and after the hydrolysis process is shown in Fig. 5. As the experimental measurements show, slightly increases in the nanofiber diameter size are observed after the hydrolysis except for HPAN-15 where the diameter decreases. The fiber roughness decreases with the diameter size and after the hydrolysis process the fibers are smooth. Hydrolyzed electrospun PAN nanofibers tend to be agglomerated, increasing this effect when the size decrease (high agglomeration at $7 \mathrm{wt} \%$ ). SEM image shows that the nanofibers are attached all together and it may results on a low water permeance through the adsorbent system. Then, obtained electrospun PAN-10 nanofibers and hydrolized ones (HPAN-10) were selected as the more appropriate support due to their diameter size (around $400 \mathrm{~nm}$ ), the apparent porosity and low agglomeration. ATR-FTIR spectra of electrospun PAN and HPAN nanofibers are shown in Fig. S3, ESI. Pristine PAN nanofibers show their characteristic peaks, while for HPAN a peak at $3396 \mathrm{~cm}^{-1}$ corresponding to the stretching of the free-hydroxyl groups and the carbonyl absorption bands with two peaks at 1718 $\mathrm{cm}^{-1}$ and $1226 \mathrm{~cm}^{-1}$ appeared, confirming the hydrolysis. Furthermore, the presence of a band at $2243 \mathrm{~cm}^{-1}$ assigned to nitrile groups indicates that not all the $\mathrm{C} \equiv \mathrm{N}$ groups were hydrolysed to $-\mathrm{COOH}$. 


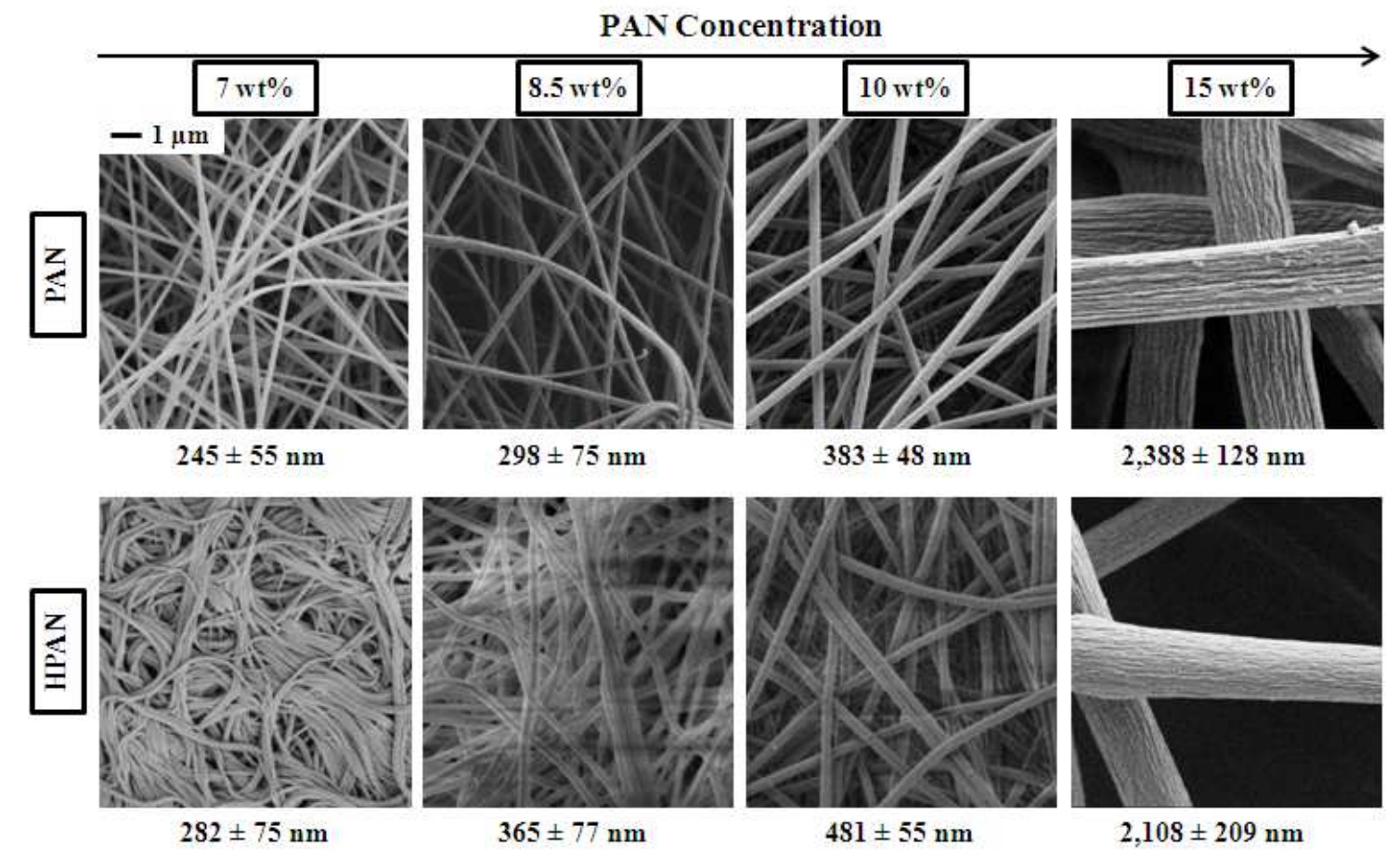

Fig. 5 SEM images and diameter distribution of PAN and HPAN nanofibers as function of PAN concentration.

\section{Characterization of SPION loaded HPAN nanofibers}

Both PAN and HPAN nanofibers were submerged in a SPION suspension for $12 \mathrm{~h}$ to test their affinity. In the case of PAN fibers no interaction with SPION is observed, resulting in a very low nanoparticle fixation, as indicated by the dark color of the suspension (see Fig. S4, ESI). On the contrary, the HPAN fibers were able to fix on their surface the majority of SPION and leaving almost transparent solution. This demonstrates that the carboxylic groups generated by hydrolysis step play a crucial role providing nanofibers with a high level of interaction needed for proper fixation of SPION. The presence of carboxylic groups on the nanofiber surface provides a corresponding ligand exchange mechanism with SPION and it produces a strong interaction that fixes the SPION on HPAN nanofibers surface. Such interaction is favored by ligand exchange mechanism, as suggests the Equation (2).

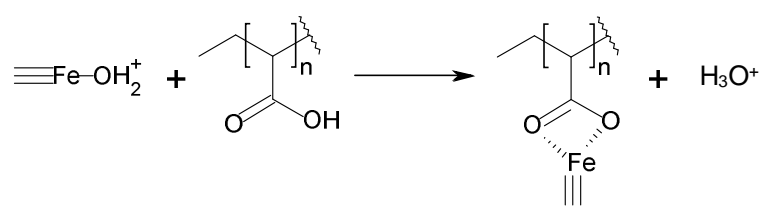

PAN-10 and HPAN-10 nanofibers where exposed to aqueous solutions with different concentrations of SPION and the amount of SPION fixed onto their surface was quantified by ICP-MS. The results in Table 1 show that the SPION fixation increases with the concentration reaching a SPION fixation higher than $97 \%$ onto electrospun HPAN nanofiber surface at SPION concentration of $144.1 \mathrm{mg}$ SPION/g HPAN. In the same conditions, only a $7 \%$ of the total SPION was fixed of the electrospun PAN nanofibers. This fact indicates that it is possible to control the SPION load by adjustment of the appropiate nanoparticles quantity in the suspension.

TEM was used to check the distribution of SPION over HPAN-10 nanofiber surface and, as shown in Fig. 6, in sample HPAN-10SPION, SPION tends to be slightly aggregated.

\section{Adsorption experiments with SPION loaded HPAN nanofibers in batch mode}

Experiments in batch mode were performed in order to test the adsorption capacity of the different adsorbent systems developed. The influence of both nanofiber diameter and SPION loading (going from 1 to $144 \mathrm{mg} / \mathrm{g}$ of nanofiber) on the total arsenate adsorption was tested under optimal conditions of contact time (60 $\mathrm{min}$ ) and pH (3.8-4.0), ${ }^{29}$ as shown in Fig. 7.

In general, compared with previous reported results with SPION in suspension (0.92 mmol As/g), ${ }^{28}$ all tested nanofiber-supported systems show superior As adsorption capacity across the whole range of SPION concentrations. SPION loaded HPAN nanofibers present a lower adsorption capacity at high SPION content, between 36.0 and $144.1 \mathrm{mg}$ SPION/g HPAN, probably due to nanoparticle aggregation onto the nanofiber surface, decreasing the specific surface area and hence the adsorption capacity. Then, below $40 \mathrm{mg}$ of SPION/g of fibers the uptake capacity increases steadily reaching a maximum around $2.9 \mathrm{mg}$ SPION/g HPAN. This value is the optimal loading, as further reduction of SPION concentration leads to a considerable decline in performance.

Table 2 shows the maximum adsorption capacities of the different nanofiber-based adsorbents developed in this work. The highest 
Table 1. Comparison between the initial SPION in suspension and the SPION fixed over PAN-10 and HPAN-10 nanofibers (Calculated assuming that all iron is in SPION form).

\begin{tabular}{cccc}
\hline Sample & $\begin{array}{c}\text { SPION in } \\
\text { solution } \\
\text { (mg/L) }\end{array}$ & $\begin{array}{c}\text { SPION on } \\
\text { nanofibers } \\
\text { (mg/g) }\end{array}$ & $\begin{array}{c}\text { SPION } \\
\text { incorporation } \\
\text { (\%) }\end{array}$ \\
\hline $\begin{array}{c}\text { PAN-10- } \\
\text { SPION }\end{array}$ & 144.1 & 10.1 & 7.0 \\
\hline & 144.1 & 139.8 & 97.7 \\
& 72.7 & 71.0 & 97.0 \\
HPAN-10- & 17.3 & 34.5 & 95.0 \\
SPION & 8.6 & 15.8 & 91.3 \\
& 5.8 & 7.3 & 84.9 \\
& 3.5 & 4.9 & 84.5 \\
& 1.4 & 2.9 & 82.9 \\
\hline
\end{tabular}
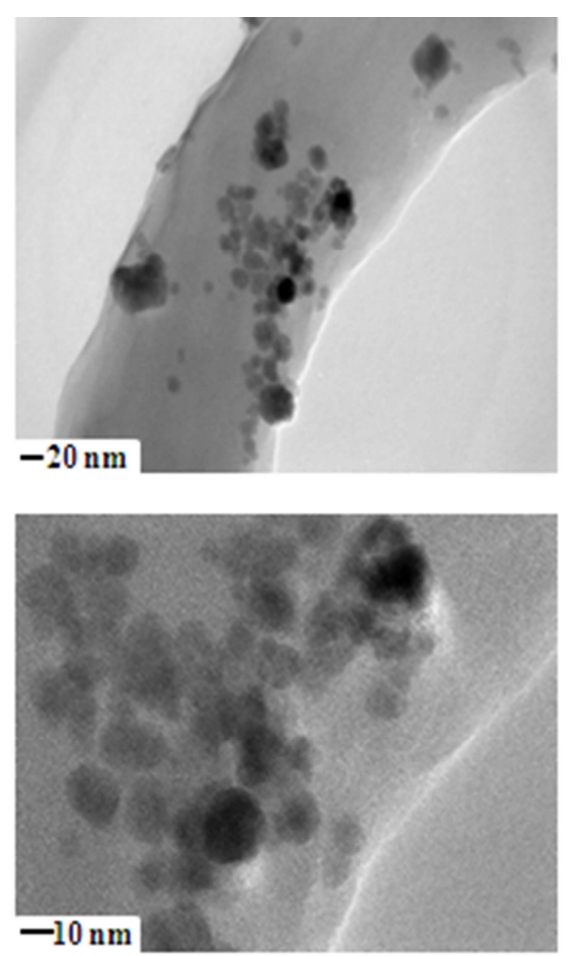

Fig. 6 TEM image of the HPAN-10-SPION nanofibers.

values obtained with the HPAN-10-SPION showed a maximum uptake capacity of $32.5 \mathrm{mmol}$ As/g SPION. Such results are 36 times higher than the results obtained with SPION in suspension under the same experimental conditions. Therefore, clearly demonstrates that nanofibers play a crucial role in amending nanoparticle

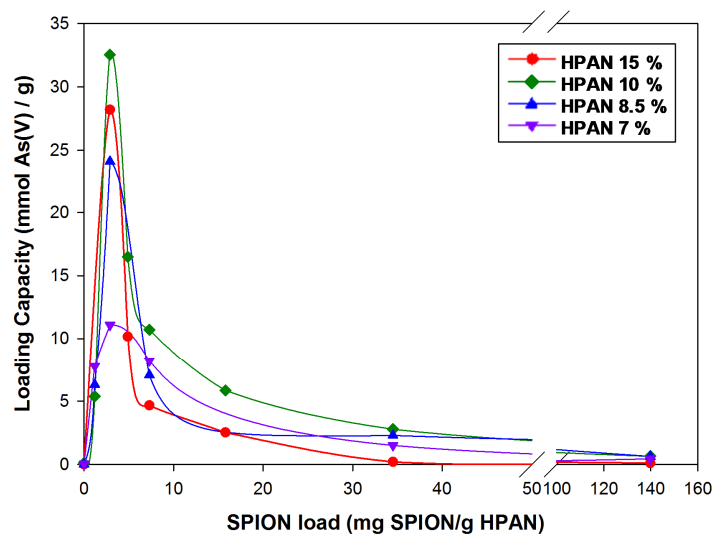

Fig. 7 Adsorption capacity, expressed in mmol of $A s(V)$ per gram of SPION, for the different nanofiber based adsorbent systems and for unsupported SPION.

aggregation keeping the SPION properties intact. Moreover, the nanofiber-based materials exceed our previously published work where SPION was supported onto a commercial cellulosic sponge $\left(\right.$ Forager $\left.^{\circledR}\right) .{ }^{29}$ It is noteworthy to mention that both pristine PAN and HPAN nanofibers present negligible arsenate adsorption (Table S3, ESI). Hence, PAN and HPAN nanofibers act just a support for SPION and do not interfere in the adsorption process.

The overall uptake capacity of fibrous materials is driven by two main competing factors. On one hand, decreasing the fiber diameter leads to larger specific surface area for SPION dispersion and hence to higher arsenate adsorption. On the other hand, as shown in Fig. 8, smaller fibers tend to cluster after hydrolysis which makes the material less porous decreasing the available contact area and generating preferential channels limiting the SPION-As interaction. For instance, HPAN-7-SPION in spite of having the smaller fiber diameter of $239 \pm 20 \mathrm{~nm}$, resulted in the the lowest adsorption capacity of $11.1 \mathrm{mmol}$ As/g SPION due to severe nanofiber aggregation.

Fibers clustering is less pronounced in larger fibers, with samples HPAN-8.5-SPION $(368 \pm 31 \mathrm{~nm})$ and HPAN-10-SPION $(465 \pm 39 \mathrm{~nm})$ adsorbing, respectively, 24.1 and $32.5 \mathrm{mmol}$ As/g SPION. Then, for larger fibers such as HPAN-15-SPION $(2380 \pm 46 \mathrm{~nm})$, the specific surface area becomes the dominant factor causing a decrease in adsorption capacity to $28.2 \mathrm{mmol}$ of As/g of SPION.

In addition, HPAN-10-SPION nanofibers present high swelling capacity, (See Fig. S5, ESI) as $100 \mathrm{mg}$ of nanofibers in contact with water can expand to occupy a volume of about $500 \mathrm{~mL}$. This behaviour plays an important role in the adsorption process. The swelling capacity generates a higher contact surface area of the nanofibers improving the interaction between As(V) and SPION and then, the arsenic adsorption capacity. 

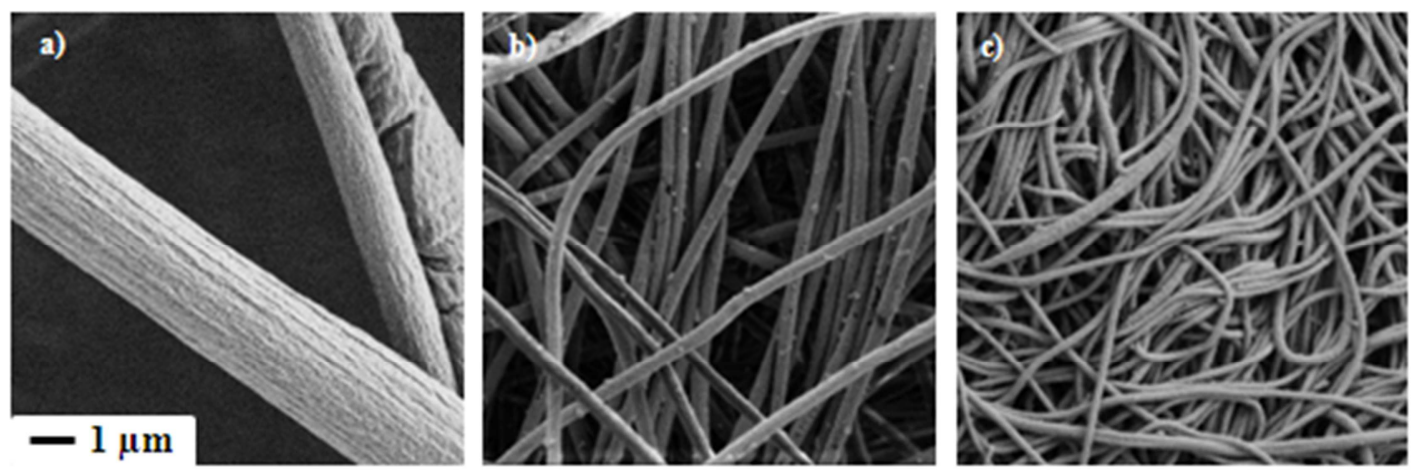

Fig. 8 SEM image of HPAN-15-SPION (a), HPAN-10-SPION (b) and HPAN-7-SPION nanofibers loaded with 2.9 mg SPION/g HPAN nanofiber.

Table 2. Adsorption capacity comparison, in batch mode, for different adsorbent systems. (SPION loading onto nanofibers was $2.9 \mathrm{mg} / \mathrm{g}$ ).

\begin{tabular}{cc}
\hline Sample & $\begin{array}{c}\text { Maximum adsorption capacity } \\
\text { (mmol As(V)/g SPION) }\end{array}$ \\
\hline HPAN-7-SPION & 11.1 \\
\hline HPAN-8.5-SPION & 24.1 \\
\hline HPAN-10-SPION & 32.5 \\
\hline HPAN-15-SPION & 28.2 \\
\hline SPION in suspension & $0.9^{29}$ \\
\hline Sponge-loaded SPION & $12.1^{29}$ \\
\hline
\end{tabular}

Ensuring the absence of nanoscale solids in the purified streams is critical as nanotechnology based products must comply with strict environmental regulations during both their use and disposal after effective life cycle. Nanoparticles released during the treatment process may pose a health risk, as the toxicity effect to the end user is not well known. To assess the stability of SPION in the experimental media, the iron content in the supernatant phase was also quantified by ICP-MS. In all samples, the results reveal iron content below the detection limit of the ICP-MS equipment ( $1 \mathrm{ppb}$ ). This indicates that the interaction between HPAN nanofibers and SPION is strong enough to prevent nanoparticles to be released during adsorption experiments, eliminating the risk of secondary contamination of the treated media. Hence, expensive extra stages for the separation of potentially released nanoparticles, such as nanofilters or magnetic separators, might not be needed.

\section{Adsorption experiments with SPION loaded HPAN nanofibers in continuous mode}

Once the optimum size and the maximum adsorption capacity were determined in batch mode, adsorption experiments in continuous mode were performed to observe the behavior of the adsorbent system under real working conditions. Gravity assisted adsorption experiments were carried out for HPAN nanofibers with both maximum (144.1 mg SPION/g HPAN) and optimal (2.9 mg SPION/g HPAN) SPION load to verify that the developed materials present the same behavior than in batch mode. As shown in Fig. 9a, while blank (HPAN-10 nanofibers) and HPAN-10-SPION nanofibers with maximum SPION load present a negligible adsorption capacity, HPAN-10-SPION nanofibers with optimal load present high adsorption capacity reaching to $52.6 \mathrm{mmol}$ As(V) per gram of SPION. Such value is almost twice the obtained one working in batch mode with the same sample. HPAN-10-SPION with maximun SPION load present a negligeable As(V) uptake as result of the high aggregation of SPION. Nanoparticle agglomeration produces a decrease in their available surface area for the interaction with arsenic leading to a very low adsorption capacity. However, SPION loaded HPAN nanofibers compression by the gravity adsorption procedure becomes a limitation which is higher when the SPION concentration increases. As shown by Fig. 9b, the SPION loaded HPAN nanofibers are saturated in a very short period of time (10 min). The compression of the composite material results in a reduction of both the available surface area and the contact time with the solution. This leads to a dramatic decrease of the adsorption capacity and to a premature saturation as some of the active centers are inaccessible to interact with arsenate during the adsorption process

To highlight the dynamic behavior of the system, Fig. 10 collects the different experiments in form of break through curves. While the adsorption in gravity mode reach up to $20.25 \mathrm{mmol} \mathrm{As}(\mathrm{V}) / \mathrm{g} \mathrm{SPION}$, the adsorption in counterflow reaches an adsorption capacity around $64.5 \mathrm{mmol} \mathrm{As}(\mathrm{V}) / \mathrm{g} \mathrm{SPION}$. As it was expected, the counterflow mode experiments present a different profile than gravity experiments, avoiding preferential channels, improving the contact between the arsenic ions and the SPION and producing higher efficiency for As(V) adsorption. It is noteworthy that SPION loaded HPAN nanofibers are not compressed during the counterflow experiment. This fact solves the limitations previously observed in the adsorption process, allowing HPAN-10-SPION to express his full potential, with the adsorption capacity reaching the extremely high value of $851.7 \mathrm{mg}$ of $\mathrm{As}(\mathrm{V}) / \mathrm{g}$ of adsorbent system.

At the end of their life cycle, saturated nanoparticles from water treatment are a highly toxic solid waste that needs to be safely disposed against future leakage of pollutants. ${ }^{37}$ The best way to reduce the potential environmental effects of nanoparticle disposal is to minimize their amount used in the wastewater treatment. In this sense, supporting SPION on nanofibers has shown to drastically improve their arsenic up-take, therefore a much smaller amount of 

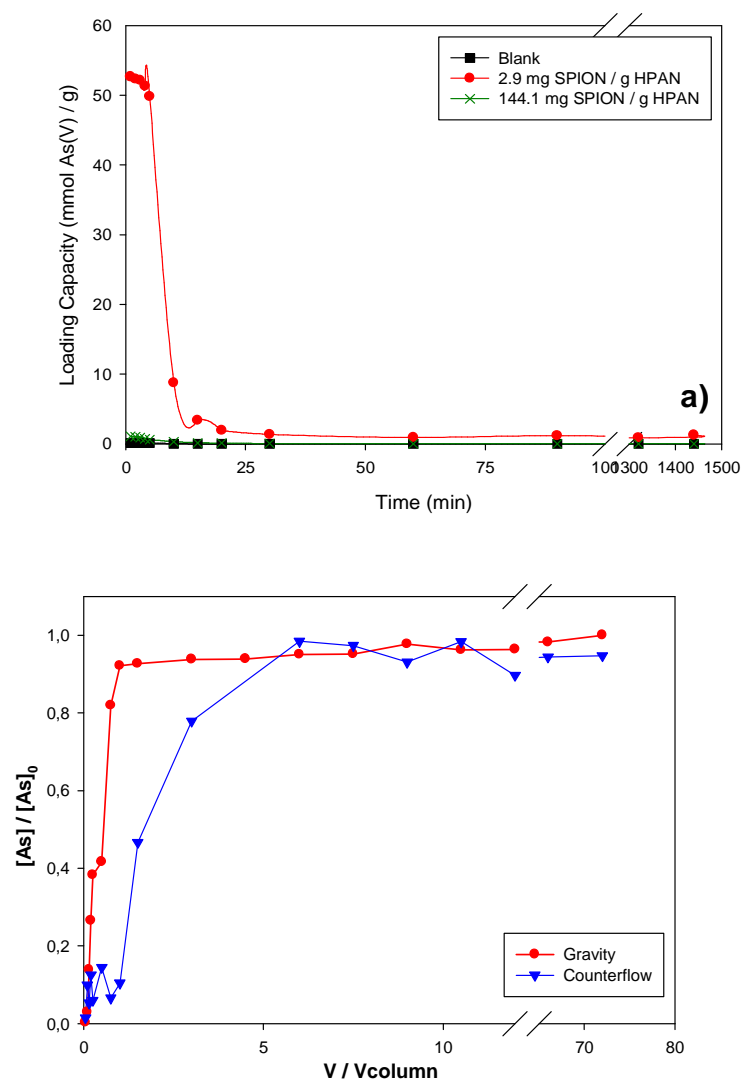

Fig. 10 Break-through curves for As(V) adsorption with HPAN$10-$ SPION by gravity and counterflow modes.

nanoparticles is needed to achieve the same efficiency. In addition, spent nanofiber material can be compressed generating a small volume of solid waste that can be easily treated, managed or stored. This renders less cost for waste disposal making the overall treatment economically competitive.

\section{Adsorption experiments with SPION loaded HPAN nanofibers in real wastewater matrix}

To assess the system performance under real conditions, adsorption experiments were performed treating dumping lixiviation wastewater doped with $\mathrm{As}(\mathrm{V})$. Such test allowed to evaluate the adsorbent system sustainability for its application when industrially contaminated water samples are treated.

A comparison between real wastewater samples and synthetic solutions using counterflow mode adsorption process was performed to characterize the adsorbent system performance against real matrices. As Fig. 11 shows, the adsorption curves for both synthetic and real solutions are similar at the very beginning of the adsorption process. Synthetic water presents a faster saturation than real wastewater showing small differences in the $\mathrm{As}(\mathrm{V})$ removed percentage. Moreover, the presence of Fluoride and Chloride ions in the real wastewater matrix does not interfere in the adsorption process. Fluorides and chlorides are non oxoanions and their interfering effect in the ligand exchange process is low compared with nitrate, sulphate of phosphate anions. Its noteworty to say that, during the first hour of operation, the column is capable of removing all $\mathrm{As}(\mathrm{V})$ that is present in the wastewater.

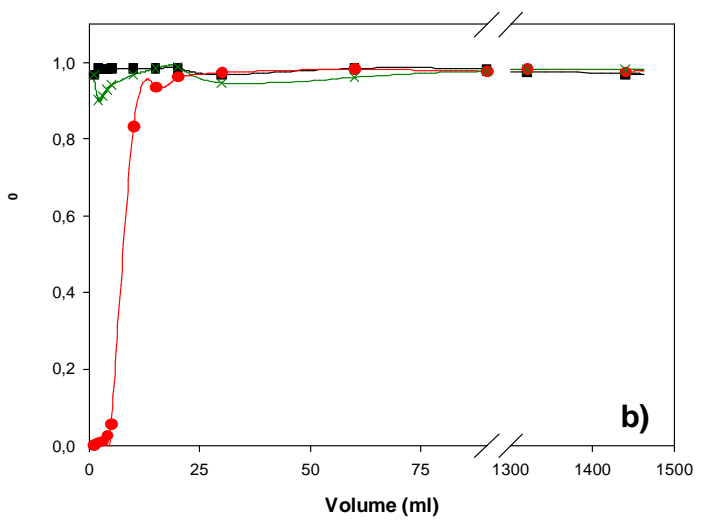

$\operatorname{rrp1}$

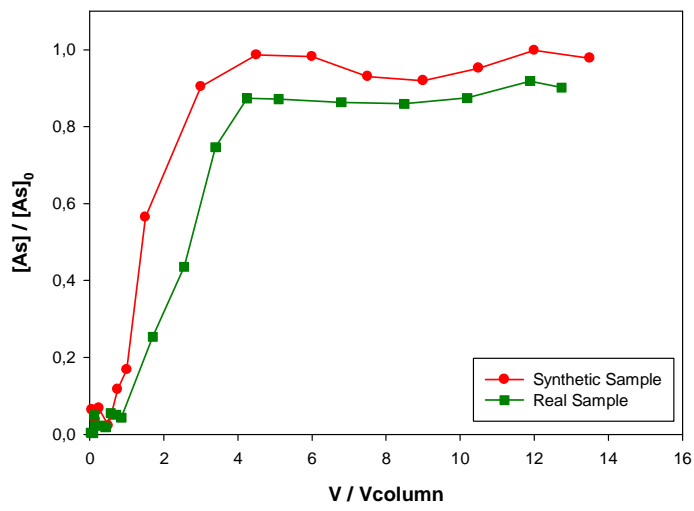

Fig. 11 Break-through curves comparison of real and synthetic samples in counterflow mode.

\section{Conclusions}

In summary, higly efficient arsenic adsorbents were fabricated by a straigh-forward and scalable process consisting of electrospinning of PAN nanofibers followed by inexpensive hydrolysis and SPION impregnation steps in aqueous media. The As(V) removal using SPION loaded nanofibers was was found to be highly dependent on both fiber diameter size and the SPION loading. Remarkably, the maximum uptake capacity was achieved with an extremely low loading of only $2.9 \mathrm{mg}$ of SPION/g of adsorbent. Supporting on nanofibers has shown to be a viable strategy to dramatically improve SPION uptake capacity up to 36 times compared with nanoparticles in suspension. The nanocomposites also demonstrated excellent performance when tested in a continuous flow system with real indutrial wastewater. Finally, no nanoparticles leaching was observed and the material is highly compressible reducing the end-of-life costs of handling and disposal. It is expected that the novel adsorbents have great potential in arsenic removal from polluted water.

\section{Acknowledgements}

The financial support of this work was provided by European Community's Seventh Framework Programme (FP7-INCO-2013-9) under Grant Agreement no. 609550 of the project named FP4BATIW and by the Innovation Found for Competitiveness of the Chilean Economic Development Agency (CORFO) under Grant no. es 13CEI2-21839. 


\section{References}

1 T. Viraragharan, K. S. Subramanian, J. A. Aruldoss, Water Sci.Technol., 1999, 40(2), 69-76.

2 S. Lata, S. R. Samadder, J. Environ. Manege., 2016, 166, 387406.

3 P.L. Smedley and D.G. Kinniburgh, Appl. Geochemistry, 2005, 17, 517-568.

4 I. Ali, Chem. Rev. 2012, 112, 5073-5091.

5 T. S. Y. Choong, T. G. Chuah, Y. Robiah, F. L. G. Koay, I. Azni, Desalination 2007, 217, 139-166.

6 B. K. Mandal, K. T. Suzuki, Talanta 2002, 58, 201-235.

7 D. Mohan, C. U. Pittman, J. Hazard. Mater. 2007, 142, 1-53.

8 D. K. Nordstrom, Science 2002, 296, 2143-2145.

9 W.R. Penrose, CRC Crit. Rev. Environ. Control, 1974, 4, 465482

10 USEPA, 2010. Arsenic in drinking water. U.S. Environmental Protection http://water.epa.gov/lawsregs/rulesregs/sdwa/arsenic

11 J. Cui, Y. Du, H. Xiao, Q. Yi and D. Du, Hydrometallurgy, 2014, $146,169-174$

12 N. Valcarcel and A. Gómez, Técnicas Analíticas de Separación, Reveré S.A., Spain, 1990.

13 O. V. Kharissova, H. V. Rasika Dias, B. I. Kharisov, RSC Adv. 2016, 5, 6695-6719.

14 J. M. McNeil and D. E. McCoy, Standard Handbook of hazardous waste Treatment and Disposal, McGraw Hill Book Company, USA, 1999, p. 6.3 .

15 T. Tsakalakos, NATO Sci. Ser., II Math. Phys. Chem. (Nanostruct. Synth., Funct. Prop. Appl.), 2003, 1, 128.

16 T. Tuutijarvi , J. Lu, M. Sillanpaa , G. Chen, J. Hazard. Mater. 2009, 166, 1415-1420.

17 J. Yang, H. Zhang, M. Yu, I. Emmanuelawati, J. Zou, Z. Yuan and C. Yu, Adv. Funct. Mater., 2014, 24, 1354-1363.

18 K. Simeonidis, S. Mourdikoudis, E. Kaprara, M. Mitrakas, L. Polavarapu Environ. Sci.: Water Res. Technol., 2016, 2, 4370.

19 Z. X. Wu, W. Li, P. A. Webley, D. Y. Zhao, Adv. Mater. 2012, 24, 485-491.

20 M. Baikousi, A. B. Bourlinos, A. Douvalis, T. Bakas, D. F. Anagnostopoulos, J. Tucek, K. Safarova, R. Zboril, M. A. Karakassides, Langmuir 2012, 28, 3918-3930.

21 X. P. Dong, H. R. Chen, W. R. Zhao, X. Li, J. L. Shi, Chem. Mater. 2007, 19, 3484-3490.

22 A. H. Lu, J. J. Nitz, M. Comotti, C. Weidenthaler, K. Schlichte, C. W. Lehmann, O. Terasaki, F. Schuth, J. Am. Chem. Soc 2010, 132, 14152-14162.

23 A. Greiner and J. H. Wendorff, Angew. Chem. Int. Ed. Engl., 2007, 46, 5670-703.

24 D. Li and Y. Xia, Adv. Mater., 2004, 16, 1151-1170.

25 C. J. Luo, S. D. Stoyanov, E. Stride, E. Pelan and M. Edirisinghe, Chem. Soc. Rev., 2012, 41, 4708-35.

26 J. Sutasinpromprae, S. Jitjaicham, M. Nithitanakul, C. Meechaisue and P. Supaphol, Polym. Int., 2006, 55, 825-833.

27 D. Morillo, A. Uheida, G. Pérez, M. Muhammed and M. Valiente, J. Colloid Interface Sci., 2015, 438, 227-234.

28 H. Zhang, H. Nie, D. Yu, C. Wu, Y. Zhang, C.J.B. White, L. Zhu. Desalination, 2010, 256, 141-147.

29 D. Morillo, G. Pérez and M. Valiente, J. Colloid Interface Sci., 2015, 453, 132-141.

30 H. J. Shipley, S. Yean, A. T. Kan and M. B. Tomson, Environ. Toxicol. Chem., 2009, 28, 509-15.

31 A. Uheida, G. Salazar-Alvarez, E. Björkman, Z. Yu and M. Muhammed, J. Colloid Interface Sci., 2006, 298, 501-507.

32 J. S. Gunasekera, B. V. Mehta, A. B. Chin and I. I. Yaacob, J. Mater. Process. Technol., 2007, 191, 235-237.

33 JCPDS, International Center for Power Diffraction Data: Swarthmore, PA, Card No, 19-629 (1989).
34 F. Liu, P. Cao, H. Zhang, J. Tian, C. Xiao, C. Shen, J. Li and H. Gao, Adv. Mater., 2005, 17, 1893-1897.

35 L. A. García-Cerda, O. S. Rodríguez-Fernández, R. BetancourtGalindo, R. Saldívar-Guerrero and M. A. Torres-Torres, Superf. y Vacío, 2003, 16, 28-31.

36 C. Han, D. Zhao, C. Deng and K. Hu, Mater. Lett., 2012, 70, 70-72.

37 K. Yin, I. M. C. Lo, H. Dong, P. Rao and M. S. H. Mak, J. Hazard. Mater., 2012, 227-228, 118-125. 